\title{
BMJ Open Evaluating the role of prereduction hip traction in the management of infants and children with developmental dysplasia of the hip (DDH): protocol for a systematic review and planned meta- analysis
}

\author{
Sarah Walton, ${ }^{1}$ Emily Schaeffer, ${ }^{2,3,4}$ Kishore Mulpuri, ${ }^{2,3,4}$ Peter Cundy, ${ }^{5,6}$ \\ Nicole Williams ${ }^{5,6}$
}

To cite: Walton S, Schaeffer E, Mulpuri $\mathrm{K}$, et al. Evaluating the role of prereduction hip traction in the management of infants and children with developmental dysplasia of the hip (DDH): protocol for a systematic review and planned meta-analysis. BMJ Open 2018;8:e019599. doi:10.1136/ bmjopen-2017-019599

- Prepublication history for this paper is available online. To view these files, please visit the journal online (http://dx.doi. org/10.1136/bmjopen-2017019599).

Received 13 September 2017 Revised 16 November 2017 Accepted 28 November 2017

Check for updates

For numbered affiliations see end of article.

Correspondence to Dr Sarah Walton; sarahmbwalton@googlemail. com

\section{ABSTRACT}

Introduction Developmental dysplasia of the hip (DDH) affects 4-6 per 1000 live births in developed countries. Effective treatment to realign the hip is necessary to avoid long-term morbidities and maximise functional outcome. Treatment options depend on patient age but typically involve hip bracing and/or reduction under general anaesthetic. Some centres also employ prereduction hip traction. Historical papers suggest traction reduces risk of avascular necrosis (AVN) femoral head and reduces requirement for open reduction. However, several studies including a large retrospective cohort study, dispute this. We propose to perform the first systematic review and meta-analysis to clarify the value of prereduction hip traction in the management of DDH in children under the age of 3 years by identifying whether it impacts on the rate of successful closed reduction (CR) and risk of AVN.

Methods and analysis We will search MEDLINE, EMBASE and the Cochrane Central Register of Controlled Trials to identify potentially relevant studies. Studies reporting on incidence of successful CR, AVN femoral head and complications associated with prereduction hip traction in children of less than 3 years with DDH will be eligible for inclusion. Only randomised controlled trials, prospective and retrospective case-control and comparative cohort studies will be included in quantitative review. There will be no study design restrictions for inclusion in qualitative review. Following study selection, full-text paper retrieval, data extraction and synthesis, studies will be assessed for risk of bias and heterogeneity. If the included studies are sufficiently homogeneous, then we will perform meta-analysis. A narrative synthesis of the systematic review's results will also be presented.

Ethics and dissemination Formal ethical approval is not required as primary patient data will not be collected. The systematic review's results will be disseminated through a peer-reviewed publication.

Trial registration number CRD42017064254; Preresults.
Strengths and limitations of this study

- This is the first systematic review to investigate the impact of prereduction hip traction on clinical outcomes in developmental dysplasia of the hip, and its results should help guide clinical management decisions or will be hypothesis generating for future prospective comparative studies.

- The proposed systematic review and meta-analysis will follow the Preferred Reporting Items for Systematic Reviews and Meta Analyses guidelines, ensuring uniform structure and reporting within the review.

- Two reviewers will screen for study eligibility and perform the risk of bias assessment, minimising the chance of reviewer-based bias in the systematic review.

- A limitation of the study is that we will only include articles reported in the English language.

\section{INTRODUCTION}

Background

The term 'developmental dysplasia of the hip' (DDH) encompasses a range of conditions in which the femoral head and acetabulum are misaligned, grow abnormally or both, leading to hip joint instability. Developed countries report an incidence of around 4-6 cases of DDH per 1000 live births although this depends on the definition used for DDH and the population studied. ${ }^{12} \mathrm{DDH}$ is usually detected within the first months of life through screening programmes consisting of physical examination and either universal or selective medical imaging. ${ }^{3}$ Hips that are positive for Barlow's sign (dislocatable/subluxable) and/or Ortolani's sign (dislocated and reducible), 
dislocated and irreducible, or unstable on ultrasound examination should be monitored or treated. The aim of treatment is to realign the hip joint in order to avoid associated long-term morbidities, including muscle weakness, degenerative arthritis and chronic pain, and to maximise functional outcome.

Typically, treatment for DDH in a child less than 6 months of age is with a Pavlik harness and/or a fixed abduction brace. ${ }^{4}$ If the child is over 6 months of age or if the aforementioned holding devices fail, then closed reduction (CR) under general anaesthesia followed by hip spica casting is generally attempted. ${ }^{4}$ Some surgeons/centres employ hip traction prior to CR, but there is currently no consensus on whether this prereduction hip traction is beneficial. Historical papers suggested prereduction traction was associated with lower rates of avascular necrosis (AVN) of the femoral head and reduced requirement for open reduction (OR). ${ }^{5-8}$ However, several studies including a large retrospective cohort study, dispute this. ${ }^{9-12}$

Further investigation is warranted to clarify the value of prereduction traction in the management of DDH in infants and children. By identifying whether prereduction traction impacts on the rate of successful $\mathrm{CR}$ and risk of AVN and by analysing these outcomes in different patient ages and following different traction methods, this review should help guide clinicians in making patient-specific management decisions.

\section{OBJECTIVES}

The primary aim of this systematic review is to evaluate how prereduction hip traction impacts on the likelihood of successful CR in infants and children with DDH compared with no prereduction traction.

Our secondary objectives are to evaluate how prereduction hip traction impacts on the risk of AVN of the femoral head and/or neck in infants and children with DDH compared with no prereduction traction, and to evaluate the incidence of other adverse events/complications arising from prereduction traction.

\section{METHODS}

\section{Eligibility criteria}

Studies will be selected according to the eligibility criteria given below.

\section{Study designs and report characteristics}

Only randomised controlled trials, prospective and retrospective case-control studies, and prospective and retrospective comparative cohort studies will be included in quantitative review/meta-analysis. There will be no restrictions set on the study designs eligible for inclusion in qualitative review. No geographical, publication date or publication status restrictions shall be imposed.

\section{Participants}

We will include studies on patients diagnosed with any degree of DDH (as diagnosed using any recognised diagnostic criteria) and starting treatment at less than 3 years of age. We will include studies that additionally study children over 3 years of age if the data for outcomes in those under 3 years of age are presented separately. Due to possible discrepancies in DDH definition usage, we will extract DDH definition used in individual studies where available. We will exclude studies including patients with diagnosed neuromuscular disorders unless the patients with neuromuscular disorders are reported separately to patients without neuromuscular disorders. We will also exclude studies only including patients who started treatment for DDH at over 3 years of age.

\section{Intervention}

We will include studies on prereduction hip traction using skin traction. We will include studies on overhead (Bryant's) traction and on longitudinal traction and studies that assess both. Studies of inpatient and outpatient traction will be included. We will consider including studies assessing other types of prereduction traction on a case-by-case basis. No inclusion limits will be set on prereduction traction duration or weights used in the studies. The rest of the treatment received within the studies should ideally be in keeping with standard care at the time of the study. For example, preliminary hip bracing with Pavlik harness or fixed abduction brace would be expected to have been attempted in a patient of less than 6 months of age at diagnosis and a hip spica cast (or accepted alternative) should be applied postreduction. We will exclude studies where the intervention is skeletal rather than skin traction and where traction is being used as intended definitive treatment. We will exclude studies in which non-standard or experimental treatment is being trialled in other areas of DDH management. We will exclude studies where OR is performed routinely following traction.

\section{Comparators}

We will include studies comparing patients fulfilling the participant and intervention inclusion criteria above with those fulfilling the participant inclusion criteria but who were not treated with prereduction hip traction. The rest of the treatment received by the comparator group should ideally be in line with standard care at the time the study was carried out. We will collect information on whether adductor tenotomy and/or psoas tenotomy was performed at the time of CR. We will also include studies comparing outcomes for overhead (Bryant's) traction with those for longitudinal traction. We will include studies comparing outcomes for different traction duration and patient age subgroups.

\section{Outcomes}

Studies reporting on the following outcomes will be eligible for inclusion. 


\section{Primary outcome}

- Incidence of successful CR defined as a hip that was reduced into the acetabulum at the time of the attempted CR, remained reduced and did not require repeat $\mathrm{CR}$ or $\mathrm{OR}$.

\section{Secondary outcomes}

- Incidence of AVN of the femoral head and/or neck (as defined by any recognised diagnostic methods, eg, Salter criteria) postreduction; Degree of AVN (as defined by any recognised classification method, eg, Bucholz/Ogden classification);

- Incidence of other adverse events/complications arising from prereduction traction.

If the above outcomes are reported, they will be analysed and graded. If the above outcomes are not reported, then we will analyse surrogate outcomes described below. Therefore, studies reporting on the following outcomes will also be eligible for inclusion:

- Incidence of cases going directly to OR;

- Incidence of residual subluxation postoperatively;

- Incidence of secondary procedures, for example, pelvic and femoral osteotomies;

- Incidence of acetabular dysplasia.

Outcomes will be collected as they are reported in each study. Due to possible discrepancies in outcome definitions used, we will extract definitions used in each of the studies included. We will extract outcomes in all data forms (eg, dichotomous, continuous) as they are reported within the study.

\section{Timing}

Studies will be selected for inclusion based on the length of follow-up of outcomes. The following will be used as a guide for all study designs:

- For studies examining successful CR as an endpoint, follow-up should be at least 6 months. This should help ensure follow-up encompasses hip spica cast removal and likely will capture if further operative procedures are indicated in order to achieve reduction of the hip.

- For studies examining AVN as an endpoint, follow-up should be at least 2 years. AVN secondary to reduction cannot be assessed for at least 6 months postreduction and can become apparent up to 2 years postreduction.

Assessment of residual subluxation may be made with first hip spica cast change at 6 weeks postreduction. The assessment process will be ongoing over the 3-6 months postreduction. We note that acetabular dysplasia tends to be assessed in mid-childhood and long-term outcomes should ideally include more than 20 years of radiological and functional data. However, this follow-up time frame is not included in the majority of studies.

\section{Setting}

There will be no restrictions on the type of setting.

\section{Language}

We will include articles reported in the English language. A list of possibly relevant titles in other languages will be provided as an appendix.

\section{Search strategy}

Literature search strategies will be developed using medical subject headings and text words related to $\mathrm{DDH}$ and hip traction. We will search the following electronic bibliographic databases: MEDLINE (OVID interface, 1948 onwards), Embase (OVID interface, 1980 onwards), and the Cochrane Central Register of Controlled Trials (Wiley interface, current issue). The electronic database search will be supplemented by searching for trial protocols through metaRegister (http://www.controlled-trials. $\mathrm{com} / \mathrm{mrct} /$ ). To ensure literature saturation, we will scan the reference lists of included studies or relevant reviews identified through the search. We will circulate a bibliography of the included articles to the systematic review team, as well as to DDH experts identified by the team. Searches will be rerun just before the final analysis and further studies retrieved for inclusion. We will include articles reported in the English language. A list of possibly relevant titles in other languages will be provided as an appendix. There will be no date restrictions.

Only studies in humans will be sought. No other study design, date or language limitations will be imposed on the search, although only studies originally written in English or that have been translated into English will be included due to resource limitations. MEDLINE, Embase and the Cochrane Central Register of Controlled Trials will be searched. The specific search strategies will be created by a Health Sciences Librarian with expertise in systematic review searching. The MEDLINE strategy will be developed with input from members of the systematic review team. If possible, the search strategy will be peer-reviewed by a second Health Sciences Librarian. The draft MEDLINE search strategies for each search question are shown below. After the MEDLINE strategy is finished, it will be modified to the syntax and subject headings of the other two databases. The International Clinical Trials Registry Platform (ICTRP) Search Portal and ClinicalTrials.gov will be searched for current and recent trials, and PROSPERO will be searched for current or recently completed systematic reviews.

\section{Study records}

\section{Data management}

Systematic review data management software will not be used due to resource limitations. However, literature search results will be uploaded to EndNote, a citation manager, to facilitate sharing of references between reviewers and to help identify and remove duplicate references. In addition to this, in order to identify multiple publications on the same study and thus avoid double counting, we will compare author names, study location, sample size, prereduction traction characteristics and outcomes reported. Where multiple publications of 
the same study are found, we will compare the reports, looking for inconsistencies that might indicate study limitations. The systematic review team will develop screening and data extraction questionnaires based on inclusion and exclusion criteria and the Cochrane Consumers and Communication Review Group's data extraction template, respectively. Both questionnaires will be incorporated into a Google form. The data inputted on each Google form will be automatically transferred onto a spreadsheet summary of the results to be shared among all reviewers.

\section{Data selection}

Two reviewers (SW and NW) will independently screen titles and abstracts yielded by the electronic search against the set inclusion and exclusion criteria. Studies that appear to be relevant will be read in full by both reviewers and a conclusion made as to whether the article should be included in the systematic review. The screening questionnaire section of the aforementioned Google form will be completed during this process, serving as a record of screened study characteristics and documenting rationale for excluding studies. Where there is discrepancy in conclusions made by the two reviewers, and this cannot be resolved through discussion, a senior reviewer (PC or KM) will be consulted for their opinion. None of the reviewers will be blinded to the study authors or institution. A Preferred Reporting Items for Systematic Reviews and Meta Analyses flow diagram will be created to illustrate the winnowing process.

\section{Data collection}

We will develop a data extraction questionnaire based on the Cochrane Consumers and Communication Review Group's data extraction template. We will create an instruction manual with decision rules to be used alongside the questionnaire. Two reviewers (SW and NW) will independently pilot the data extraction questionnaire on five randomly selected studies that have met screening criteria. Following this, they will discuss any necessary changes and tailor the form accordingly. Using the prepiloted form, data extraction will be performed on all included studies by one reviewer (SW). A random subset of data extracted will be verified by another reviewer (NW) for quality control purposes. We will contact trial authors via email where clarification or additional information is required during the data extraction process.

\section{Data items}

The following data will be extracted from included studies.

\section{General information on study}

Title, author, publication status, year of publication, year study was conducted, author contact details, funding source and any conflicts of interest, original language.

\section{Study methods}

Stated aim of study, study design, number of groups in the study, consumer involvement in the study (eg, in delivering patient care and in evaluating hip traction and its outcomes).

\section{Study participants}

Description (ie, infants with DDH, their parents/carers, healthcare professionals), age range and mean age of participants, at what point age is taken (ie, at DDH diagnosis, at commencement of treatment or at attempted CR), gender, geographical location of participants (including city, state and country), study setting (ie, inpatient or outpatient), methods of recruitment for study, inclusion and exclusion criteria for participation in study, any stated excluded groups, and rationale for exclusion. If available, the following data will also be collected: severity of DDH at diagnosis, diagnostic methods, DDH definition and classification criteria used, previous treatment received (ie, Pavlik Harness, fixed abduction brace), laterality of $\mathrm{DDH}$.

\section{Study numbers}

Number of patients identified as eligible for study inclusion, number of patients excluded, number of patients refusing participation in study (prospective studies only), number of patients in traction group, number of patients in control group, number of patients not completing treatment, number of patients lost to follow-up, number of patients included in the analysis for each outcome (see Outcomes and Prioritisation section for list of outcomes to be collected) and for each group; intervention and control.

\section{Intervention characteristics}

Type of prereduction traction used (ie, overhead or longitudinal), duration of traction, weights used for traction, location of traction (ie, inpatient or outpatient), further details of location (ie, type of hospital, type of ward), who performed the traction and what training was given, description of how traction was performed including materials used and observations and monitoring performed during traction, any tailoring of traction during treatment and, if so, based on what decision rules, details of any guidelines or information used to guide treatment in the study, and details of assessment of compliance with intervention. Data on whether adductor/psoas tenotomy was performed at CR and for how long a hip spica cast was applied postoperatively should also be collected in this section. For the control group: description of treatment received. For both the intervention and the control groups, we will attempt to collect data on age range and mean age of participants at commencement of the intervention in this section.

\section{Outcomes and prioritisation}

Data will be collected on the following outcomes:

- Incidence of successful CR as defined as a hip that was reduced into the acetabulum at the time of the 
attempted CR, remained reduced and did not require repeat $\mathrm{CR}$ or OR.

- Incidence of AVN of the femoral head and/or neck (as defined by any recognised diagnostic methods, eg, Salter criteria) postreduction. Degree of AVN (as defined by any recognised classification method, eg, Bucholz/Ogden classification).

- Occurrence of other adverse events arising from prereduction hip traction and their incidence.

The definition of successful CR and the criteria used for AVN diagnosis and classification of its severity will be collected. The methods used for assessing successful CR and AVN will be collected as will the timing of the assessments for these two outcomes, including the frequency and duration of assessments and follow-up. A note on direction of effect will be made in this section alongside data collected on the outcomes to help guard against errors when the data from different studies is collated on a particular outcome. We intend to refine our outcome definitions during the systematic review process based on outcomes reported in the included studies.

Data will be collected on the following secondary outcomes:

- Incidence of cases going directly to OR;

- Incidence of residual subluxation postoperatively;

- Incidence of secondary procedures, for example, pelvic and femoral osteotomies;

- Incidence of acetabular dysplasia.

For those cases going directly to OR, the rationale for this should be recorded. How residual subluxation and acetabular dysplasia were defined and graded in each study and how they were assessed for, including frequency of assessments and duration of follow-up, will be collected. Type of secondary procedure will be recorded by intervention name and incidence of each secondary procedure will be collected. We will also collect information on how patients who did not attend for planned reviews were followed up by the studies.

\section{Risk of bias individual studies}

We anticipate that our review will encompass a variety of study designs including randomised controlled trials, case-control and comparative cohort studies. Choice of risk of bias tool will depend on the study design.

For randomised controlled trials, we will use The Cochrane Collaboration's tool for assessing risk of bias. This tool includes seven domains to consider: random sequence generation, allocation concealment, blinding of participants and personnel, blinding of outcome assessment, incomplete outcome data, selective reporting and other sources of bias. We will use the Cochrane Handbook criteria to guide decision-making. Each domain will be marked as either 'high risk', 'unclear' or 'low risk.' It will not have been possible to conceal allocation or blind participants or personnel in studies of prereduction hip traction as it is an intervention that will be obviously visible to all involved in the patient's care. Thus, we anticipate studies of prereduction hip traction to automatically score as 'high risk' in these domains. Where a judgement cannot be made based on the information provided by the original paper, a judgement of 'unclear' will be made and the study authors will be contacted for further information.

For case-control and comparative cohort studies, we will use the Newcastle-Ottowa quality assessment scale for case-control and cohort studies, respectively. This generic scale will be tailored to meet our review's requirements. Studies will be assessed on the domains of selection of study groups, comparability of groups and on ascertainment of the outcome of interest. We will consider each item on the scale separately rather than assigning an overall score. The Newcastle-Ottowa Scale manual will be used in conjunction with these scales. For each assessment completed, descriptions of the study's methods relating to each point will be included to justify decisions made. Risk of bias assessment will be performed by SW (review lead) and NW (content expert) in duplicate and disagreements on scoring of individual studies will be resolved by discussion with a third reviewer, ES (methodological expert). Blinding of the reviewers to the study authors and institutions will not be performed.

\section{Data synthesis}

A narrative synthesis of the systematic review's results will be presented. In this narrative synthesis, we will describe the results from each study, focusing on each of the systematic review's objectives in turn. The characteristics of each study will be tabulated alongside the text to allow easier comparison of patient, intervention and outcome characteristics. The results from all studies will be described including those deemed at 'high risk' of bias, in anticipation of the majority of included studies automatically scoring as 'high risk' on the 'blinding of participants and personnel' domain. Studies scoring as 'high risk' in other domains will be highlighted as such in their description. Some studies, for example, case reviews will only be described in the qualitative results section.

To proceed to meta-analysis, we will need to assess homogeneity of the included studies. We will particularly examine whether the patient characteristics (eg, age and degree of DDH), the way in which traction was applied (eg, type of traction, traction duration and weights used), and outcome measures collected are sufficiently similar between included studies. If the included studies are adequately homogeneous, then a meta-analysis will be performed. If the outcome measures recorded in the studies are heterogeneous, then a meta-analysis of all included studies will not be appropriate. Instead, we would perform quantitative analysis on subsets of studies reporting on the same objective outcome.

We will use risk ratio (RR) with $95 \% \mathrm{CI}$ as a measure of treatment effect of prereduction hip traction on rate of successful CR and risk of AVN.

We will deal with missing outcome data in published reports by contacting study authors and requesting the relevant data. Where the data are not available, we will 
consider using imputation methods and undertaking sensitivity analyses to assess the potential impact of missing data on the cumulative estimate.

Clinical heterogeneity of included studies will be assessed by examining patient and intervention characteristics. Statistical heterogeneity will be assessed. If high levels exist, we will attempt to account for this by subgroup analysis.

We will use statistical software alongside guidance from the Cochrane Handbook for Systematic Reviews of Interventions to combine the outcomes from the studies. Method used for meta-analysis will depend on the results of the tests of statistical heterogeneity. A fixed-effects model will be used if the tests are not significant and a random-effects model will be used if there is significant statistical heterogeneity. If there is considerable statistical heterogeneity between studies, then a meta-analysis will not be performed and a solely qualitative summary will be presented.

\section{Metabias(es)}

To assess for selective reporting of outcomes, we will search for the study's protocol on the Clinical Trial Register at the ICTRP of WHO and compare intended outcomes on the protocol versus reported outcomes.

\section{Confidence in cumulative estimate}

The strength of evidence for our review's estimate of RR of successful CR and RR of AVN will be assessed using the systematic Grading of Recommendations, Assessment, Development and Evaluations approach. A score for quality of our evidence: high, moderate, low or very low will be allocated for both cumulative estimates.

\section{Author affiliations}

${ }^{1}$ Neonatal Unit, Addenbrooke's Hospital, Cambridge, UK

${ }^{2}$ International Hip Dysplasia Institute, Orlando, Florida, USA

${ }^{3}$ Department of Orthopaedics, University of British Columbia, Vancouver, British Columbia, Canada

${ }^{4}$ Department of Orthopaedic Surgery, British Columbia Children's Hospital, Vancouver, British Columbia, Canada

${ }^{5}$ Centre for Orthopaedic and Trauma Research, The University of Adelaide, Adelaide, South Australia, Australia

${ }^{6}$ Department of Orthopaedic Surgery, Women's and Children's Hospital, Adelaide, South Australia, Australia

Contributors NW is the guarantor. NW and PC were involved in the conception of the project. SW, NW and ES drafted the manuscript. All authors contributed to the development of the selection criteria, the risk of bias assessment strategy and data extraction criteria. SW and ES developed the search strategy. ES provided methodological expertise. PC, NW and KM provided expertise on DDH. All authors read, provided feedback and approved the final manuscript.

Funding This research received no specific grant from any funding agency in the public, commercial or not-for-profit sectors.

Competing interests None declared.

Patient consent Not required.

Provenance and peer review Not commissioned; externally peer reviewed.

Author note The Centre for Orthopaedic and Trauma Research (The University of Adelaide) is the sponsor of the review, but no funding from the Centre for Orthopaedic and Trauma Research has been received for this review.

Open Access This is an Open Access article distributed in accordance with the Creative Commons Attribution Non Commercial (CC BY-NC 4.0) license, which permits others to distribute, remix, adapt, build upon this work non-commercially, and license their derivative works on different terms, provided the original work is properly cited and the use is non-commercial. See: http://creativecommons.org/ licenses/by-nc/4.0/

(c) Article author(s) (or their employer(s) unless otherwise stated in the text of the article) 2018. All rights reserved. No commercial use is permitted unless otherwise expressly granted.

\section{REFERENCES}

1. Pollet V, Percy V, Prior HJ. Relative risk and incidence for developmental dysplasia of the hip. J Pediatr 2017;181:202-7.

2. Woodacre T, Ball T, Cox P. Epidemiology of developmental dysplasia of the hip within the UK: refining the risk factors. J Child Orthop 2016;10:633-42.

3. Anon. Clinical practice guideline: early detection of developmental dysplasia of the hip. Committee on quality improvement, subcommittee on developmental dysplasia of the hip. American Academy of Pediatrics. Pediatrics 2000;105:896-905.

4. Al-Essa RS, Aljahdali FH, Alkhilaiwi RM, et al. Diagnosis and treatment of developmental dysplasia of the hip: a current practice of paediatric orthopaedic surgeons. J Orthop Surg 2017;25:230949901771719.

5. Yamada N, Maeda S, Fujii G, et al. Closed reduction of developmental dislocation of the hip by prolonged traction. $J$ Bone Joint Surg Br 2003;85:1173-7.

6. DeRosa GP, Feller N. Treatment of congenital dislocation of the hip. Management before walking age. Clin Orthop Relat Res 1987:77-85.

7. Daoud A, Saighi-Bououina A. Congenital dislocation of the hip in the older child. The effectiveness of overhead traction. J Bone Joint Surg Am 1996;78:30-40.

8. Langenskiöld A, Paavilainen T. The effect of prereduction traction on the results of closed reduction of developmental dislocation of the hip. J Pediatr Orthop 2000;20:471-4.

9. Pospischill R, Weninger J, Ganger R, et al. Does open reduction of the developmental dislocated hip increase the risk of osteonecrosis? Clin Orthop Relat Res 2012;470:250-60.

10. Kutlu A, Ayata C, Ogün TC, et al. Preliminary traction as a single determinant of avascular necrosis in developmental dislocation of the hip. J Pediatr Orthop 2000;20:579-84.

11. Kahle WK, Anderson MB, Alpert J, et al. The value of preliminary traction in the treatment of congenital dislocation of the hip. $J$ Bone Joint Surg Am 1990;72:1043-7.

12. Sucato DJ, De La Rocha A, Lau K, et al. Overhead bryant's traction does not improve the success of closed reduction or limit AVN in developmental dysplasia of the hip. $J$ Pediatr Orthop 2017;37:e108-13. 